\title{
Tendencias del cáncer de mama en América Latina y El Caribe
}

\author{
Rafael Lozano-Ascencio, MD, M en C, , $^{(1,23)}$ Héctor Gómez-Dantés, MD, M en C, ${ }^{(3)}$ Sarah Lewis, MSc, ${ }^{(3)}$ \\ Luisa Torres-Sánchez, $\mathrm{PhD}$, ${ }^{(4)}$ Lizbeth López-Carrillo, $\mathrm{PhD} .{ }^{(4)}$
}

\begin{abstract}
Lozano-Ascencio R, Gómez-Dantés H, Lewis S,Torres-Sánchez L, López-Carrillo L. Tendencias del cáncer de mama en América Latina y El Caribe. Salud Publica Mex 2009;5 I supl 2:SI 47-SI 56.
\end{abstract}

\section{Resumen}

Objetivo. Describir la magnitud y distribución espaciotemporal del cáncer de mama (CaMa) en América Latina y el Caribe (LAC) de 1979 a 2005. El CaMa destaca como la causa de muerte más importante por tumores malignos en las mujeres latinoamericanas, incluso por arriba del cáncer cervicouterino. Material y métodos. Revisión y organización de las estadísticas nacionales e internacionales (registros, estudios publicados y bases de datos) sobre incidencia y mortalidad. Elaboración de estimadores básicos, razones de mortalidad para cada país y análisis de tendencias. Resultados. Aun con el rezago de información presente en la región, se observa un incremento de la incidencia y la mortalidad por CaMa. La razón de muertes/casos en los países de LAC muestra problemas de acceso a la detección y tratamiento. Conclusiones. El reto es mejorar los sistemas de información y la infraestructura diagnóstica para la detección oportuna y el tratamiento adecuado con la finalidad de detener la tendencia ascendente de la mortalidad prematura.

Palabras clave: tumores malignos; cáncer de mama; tendencia de mortalidad;América Latina

\section{Lozano-Ascencio R, Gómez-Dantés H,}

Lewis S, Torres-Sánchez L, López-Carrillo L.

Breast cancer trends in Latin America and the Caribbean.

Salud Publica Mex 2009;5 I suppl 2:SI 47-SI 56.

\begin{abstract}
Breast cancer is currently the most significant cause of death from malignancies in Latin American women, including cervical cancer. Objective. Describe the magnitude and spatial-temporal distribution of breast cancer in Latin America and the Caribbean (LAC) from 1979-2005. Material and Methods. National and international incidence and mortality statistics were reviewed and organized (registries, databases, and published literature), basic estimators and mortality ratios for each country were calculated and trends were analyzed. Results. Despite substantial data gaps in incidence for many countries, an increase in incidence and mortality is observed in LAC countries. Deaths/cases ratios illustrate problems in access to detection and treatment and the greatest gaps are observed in poorer countries. Conclusions. A regional effort is needed to improve information systems related to cancer in general and breast cancer in particular. It is imperative to develop strategies to improve diagnostic infrastructure in order to achieve early detection and effective treatment and halt the upward trend in premature mortality.
\end{abstract}

Key words: malignant tumors; breast cancer; mortality trends; Latin America; Caribbean

Este estudio fue posible gracias al apoyo fiinanciero de Instituto Carso de la Salud y el Consejo Promotor Competitividad y Salud de la Fundación Mexicana para la Salud.

(I) Instituto para la Métrica y Evaluación en la Salud, Universidad de Washington. Seattle, Washington, Estados Unidos.

(2) Hospital Infantil de México, Federico Gómez. México, DF.

(3) Fundación Mexicana para la Salud, Observatorio de la Salud. México, DF.

(4) Instituto Nacional de Salud Pública, Dirección de Salud Reproductiva. Cuernavaca, Morelos, México.

Fecha de recibido: 20 de noviembre de 2008 - Fecha de aprobado: 17 de diciembre de 2008 Solicitud de sobretros: Mtro. Rafael Lozano Ascencio. Institute for Health Metrics and Evaluation, University of Washington. 230I, 5th Ave. Suite 600. Seattle.WA 9812I. USA.

Correo electrónico: rlozano@u.washington.edu, lozano@prodigy.net.mx 
$\mathrm{H}^{2}$ asta 5\% de la carga de la enfermedad en el mundo se relaciona con los tumores malignos. En los países ricos, esta cifra se eleva a $14.6 \%$ y disminuye a $2.2 \%$ en los pobres. La diferente carga atribuible a los tumores malignos se vincula con dos aspectos; por un lado, el riesgo de morir por estas causas en los países ricos es 2.4 veces mayor que en los países pobres y de ingresos medios, lo cual se refleja en un mayor número de años de vida perdidos por muerte prematura por otras causas y, por el otro, se observa una diferencia significativa en la contribución de los años vividos con discapacidad. Mientras que en los países ricos la carga de los tumores malignos relacionada con discapacidad es de $11.5 \%$, en las naciones pobres es de sólo 2.6\% ${ }^{1}$ En gran medida, la mayor disponibilidad y acceso a la tecnología diagnóstica y terapéutica para curar los tumores malignos explican la magnitud de estas diferencias.

En el caso del cáncer de mama (CaMa), la situación es similar. En el mundo, alrededor de $1 \%$ de la carga de la enfermedad en las mujeres se vincula con esta causa y varía de $3.2 \%$ en los países ricos a $0.4 \%$ en los pobres. En América Latina, los años de vida saludable (AVISA) perdidos debidos al CaMa ascienden a 615 000, lo cual representa $1.4 \%$ del total de AVISA por todas las causas y una tasa de 221 por 100000 mujeres. De ese total, 91\% corresponde a años perdidos por muerte prematura y 9\% a años vividos con discapacidad. En contraste, en Estados Unidos y Canadá se pierden 673000 AVISA (3\% del total y una tasa de 410 por 100000 mujeres), pero $71 \%$ se debe a muertes prematuras y $29 \%$ a discapacidad. El riesgo de perder un año por muerte prematura por CaMa es 1.4 veces mayor para las mujeres de Norteamérica; cuando se refiere a años de vida con discapacidad, el riesgo es 6.3 veces mayor. ${ }^{1}$

El notable aumento de la supervivencia de las mujeres que padecen cáncer de mama y habitan en el norte del continente explica estas diferencias. Los resultados del estudio mundial de supervivencia a cinco años de mujeres diagnosticadas con CaMa varían de $84 \%$ en Norteamérica a menos de 65\% en Brasil y Eslovaquia. Los países europeos que participaron en el estudio poseen una sobrevida de 70 a $79 \% .^{2}$ En fecha reciente, la información disponible ubica el tema del CaMa en la agenda de salud pública, no obstante que la tendencia creciente en su morbimortalidad, tanto en países ricos como en los de recursos bajos y medios, se ha reconocido desde hace 25 años.

En los últimos años se ha documentado la importancia que el entorno social juega en la determinación de la enfermedad, así como en las variaciones de la incidencia entre diferentes áreas geográficas. ${ }^{3}$ Estudios sobre migrantes de países asiáticos a Estados Unidos de América ${ }^{4}$ o de mujeres de Polonia a Australia, ${ }^{5}$ ofre- cen sólida evidencia de que es menor el componente genético que el ambiental. El incremento del riesgo de padecer o morir por esta causa en países pobres y de ingresos medios se vincula con cambios en los patrones reproductivos y nutricionales, además de la disminución de la fecundidad, ${ }^{5}$ los cambios en los estilos de vida y las condiciones de trabajo de las mujeres. ${ }^{6}$ De manera adicional, las diferencias en el acceso a los servicios de salud y la tecnología disponible para la detección y tratamiento oportunos, ${ }^{7,8}$ así como el desempeño de los servicios de salud en la oferta de servicios de calidad, ${ }^{9}$ determinan las brechas de mortalidad y supervivencia por CaMa.

Aunque la mayoría de los factores de riesgo identificados puede ser modificable, los retos para los sistemas de salud se concentran en actividades que permitan fortalecer la prevención primaria (investigación y desarrollo de tecnologías) y actividades de prevención secundaria y terciaria. La efectividad de la detección temprana y el tratamiento adecuado depende de la oportunidad con la que se aplican; de otra manera, los costos de la atención se incrementan en grado considerable, las ganancias en salud resultan mínimas y el esfuerzo desarrollado por el sistema es poco alentador.

El padecimiento puede ocurrir en mujeres de cualquier nivel social, económico y étnico, aunque son las mujeres con mayores desventajas sociales y menores recursos las más vulnerables. ${ }^{10}$ Pese a la abundancia de bibliografía relacionada con el tema, es conveniente mantener actualizada la descripción epidemiológica sobre el CaMa, sobre todo en regiones donde el rezago en el acceso a la detección temprana y al tratamiento es un asunto común. La descripción que aquí se presenta incluye datos mundiales y la medición de los resultados en salud (morbilidad, mortalidad y letalidad) para Latinoamérica y El Caribe (LAC), con particular énfasis en la necesidad de disponer de información relevante, oportuna y comparable para mejorar la asignación de recursos y las decisiones que acompañan las políticas nacionales y regionales de prevención del CaMa.

\section{Material y métodos}

Se trata de un estudio descriptivo sobre el comportamiento de la incidencia y la mortalidad por CaMa en mujeres de 25 años y mayores residentes en 23 países de América Latina y El Caribe, los cuales representan 95\% de la población de la región. Sólo se presentan datos para las mujeres, aunque el CaMa también puede afectar a los hombres. Como criterio de inclusión se consideró contar con información por lo menos en 15 de los 26 años que se incluyeron en el estudio (1979-2005), sin tomar en cuenta el tamaño de la población. Por esta razón, en especial en 
los países de El Caribe, es importante considerar que las variaciones pueden relacionarse con la escasez de datos o un número pequeño de casos o defunciones.

Se realizó una revisión sistemática de los registros publicados y no publicados, la consulta de fuentes estadísticas y la revisión de diversas publicaciones con información para resumir la situación actual del CaMa en las mujeres latinoamericanas. Las bases de datos consultadas fueron: PubMed/Medline, GoogleScholar, bases de datos internacionales y nacionales, y bases gubernamentales de países latinoamericanos. La búsqueda se concentró en la identificación de artículos que describieran la magnitud del CaMa en la región de América Latina y El Caribe.

La incidencia por CaMa se obtuvo de los datos provenientes de la Agencia Internacional para la Investigación del Cáncer (IARC, por sus siglas en inglés) publicados en GLOBOCAN 2002. ${ }^{11}$ Las estimaciones de incidencia de GLOBOCAN en 28 países de América Latina y El Caribe se basan en el uso de las siguientes fuentes de información: registros nacionales del cáncer (Cuba, Costa Rica y Puerto Rico); datos sobre frecuencia del cáncer (Haití y Bolivia); promedios del área/región (Guatemala y Honduras); y los datos de mortalidad para el resto de los países mediante las estimaciones de la incidencia. Los registros consultados fueron los de Brasil, Campinas (1991-1995); Colombia, Cali (1992-1996); Costa Rica (1995-1996); Cuba, Villa Clara (1995-1997); y Ecuador, Quito (1993-1997). La mortalidad se calculó con base en el número de muertes por CaMa informado en las estadísticas vitales de los países miembros de la región de las Américas que concentran la Organización Mundial y Panamericana de la Salud (OMS y OPS) y las proyecciones de población (denominadores) de la División de Población de las Naciones Unidas en diferentes años. ${ }^{12}$ En ambos casos, el ajuste por edad se realizó tras considerar, como población estándar, la población mundial recomendada por la OMS. ${ }^{13}$ La tendencia en la mortalidad se analizó mediante una regresión lineal simple entre la tasa de mortalidad por CaMa ajustada por edad en cada uno de los 23 países y los años de estudio comprendidos entre 1979 y 2005.

Debido a que en la actualidad el CaMa no es susceptible de prevención primaria, la detección temprana y el tratamiento oportuno y adecuado funcionan como los elementos moderadores en la razón muertes / casos (M/C). Una razón de 1.0 significa que $100 \%$ de las mujeres diagnosticadas muere, mientras que una razón de 0 supone que ninguna de las mujeres con diagnóstico fallece. Como una medida de la efectividad y oportunidad del tratamiento sobre esta enfermedad, se estimó la M/C para cada uno de los países. Para ello se dividió el número de muertes por CaMa calculados para el año 2002 sobre el número de casos nuevos de la enfermedad estimados por GLOBOCAN para el mismo año. Con fines de comparación, se estimó la razón muerte/ caso para la región de LAC y las Américas en general; esta última incluye a Canadá y Estados Unidos de América. Para calcular la incidencia y la razón M/C en LAC, se promedió la información correspondiente a los 28 países de Sudamérica, Centroamérica y El Caribe considerados en el GLOBOCAN 2002. Para la región de las Américas se incluyó además la correspondiente de Estados Unidos y Canadá.

\section{Resultados}

\section{Morbilidad}

Cada año se diagnostican alrededor de 10 millones de casos de tumores malignos en hombres y mujeres en todo el mundo. De los casi 6 millones de tumores malignos que ocurrieron en las mujeres en el año 2007, el CaMa se ubicó en el primer lugar con 1.3 millones, $27 \%$ de ellos en países desarrollados y $19 \%$ en países en desarrollo. ${ }^{14}$ El incremento del número de casos nuevos entre 2002 y 2007 en los países en desarrollo fue dos veces mayor que el observado en los países desarrollados. En LAC se registran cerca de 100000 casos anuales con un incremento durante el periodo mencionado de $18 \%$, con una variación desde 10\% en El Caribe hasta 21\% en Centroamérica.

La incidencia del CaMa va en aumento en todos los países del mundo y se reconoce una diferencia de 10 veces en el riesgo de desarrollar este padecimiento entre las regiones con mayor y menor incidencia. Según la regionalización de IARC, la tasa más alta en 2002 procede de Sudamérica con 46, seguida por El Caribe con 32.9 y Centroamérica con 25.9 por 100000 mujeres. Como se advierte en la figura 1, la tasa más baja se registró en Haití con 4.4 casos por 100000 mujeres, lo cual contrasta con los países del cono sur donde la incidencia es de 75 y 83 por 100000 mujeres en Argentina y Uruguay respectivamente, que son similares a las observadas en Estados Unidos de América y Canadá.

\section{Mortalidad}

De acuerdo con las previsiones de la OMS para 2004, $31 \%$ de las defunciones por CaMa ocurrió en países de ingresos altos, $44 \%$ en las naciones de ingresos medios y $24 \%$ en los países pobres. En LAC, el CaMa representó $18 \%$ del total de las defunciones por tumores malignos y $3 \%$ del total de muertes en las mujeres. En Barbados, 


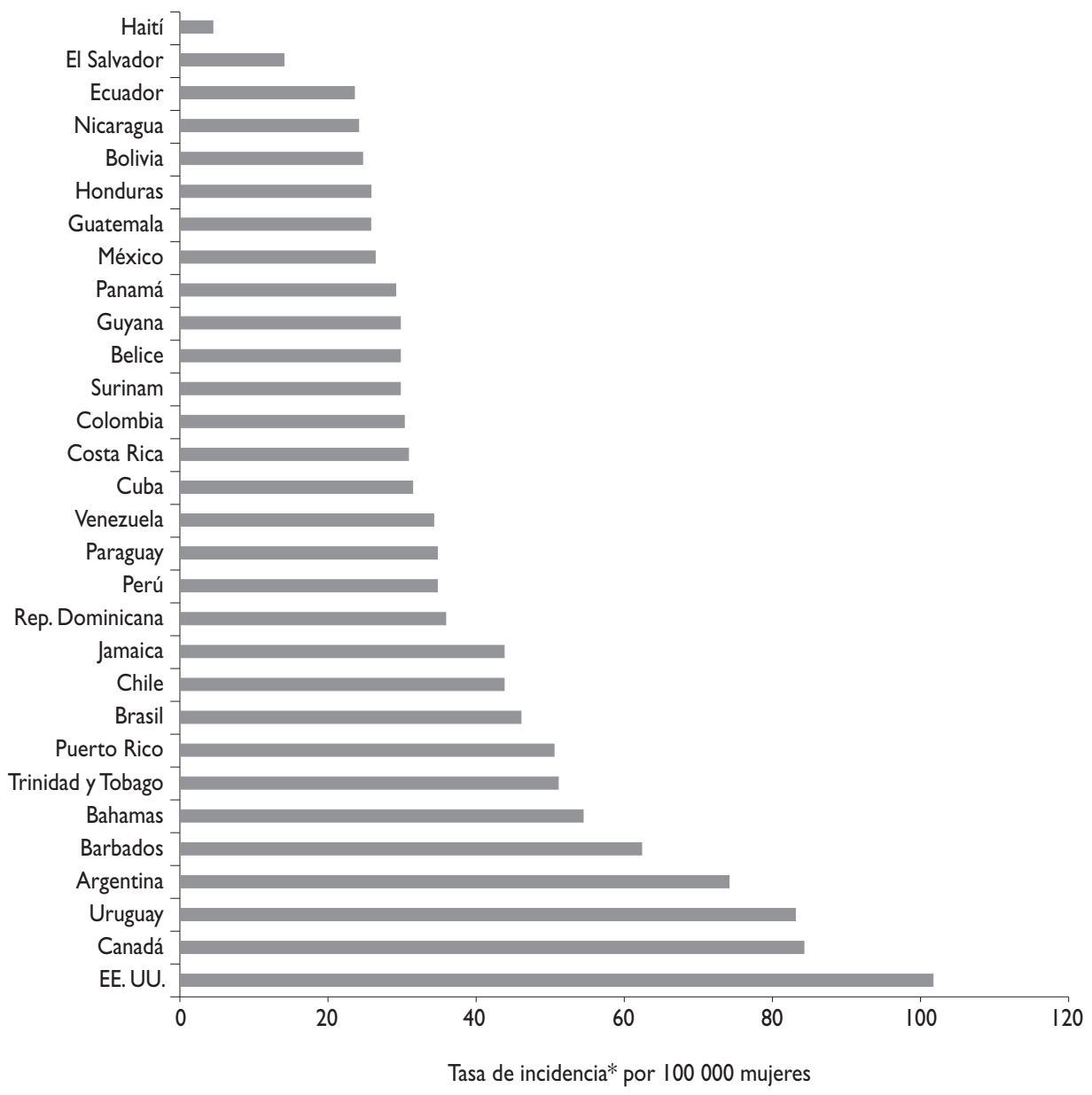

Fuente: IARC, GLOBOCAN 2002 (tasa ajustada por edad según el estándar mundial a través de grupos de edad, 0-14,15-44,45-54,55-64,65+)

\section{Figura I. InCidencia* del CÁncer de mama en países de América, 2002}

Bahamas, Trinidad y Tobago, Argentina y Uruguay entre 20 y $25 \%$ de la muertes por neoplasias malignas en las mujeres se debió a dicha neoplasia. Por su parte, la tasa de mortalidad por CaMa ajustada es de tres a cuatro veces menor en los países de ingreso más bajo respecto del alto (10.6 contra 32.3 defunciones por 100000 mujeres); tal es el caso de LAC, con una tasa de 17 por 100 000, que es menor a las tasas observadas en Norteamérica (31.6) o Europa Occidental (42.5).

Cifras recientes (2005) muestran que la mortalidad por CaMa, en los países de El Caribe inglés y el Cono Sur, es la más alta en la región de LAC, en contraste con la que se registra en Centroamérica. El riesgo de morir en las mujeres que viven en Bahamas, Barbados, Trinidad y Tobago, Argentina, Cuba y Uruguay es cuatro a cinco veces más alto que en Salvador, Nicaragua, Guatemala y México (figura 2).*

Al analizar la tendencia de 1979 a 2005 en los países de la región se observan tres grupos: a) los países donde ha disminuido la mortalidad (Argentina, Uruguay y Bahamas);b) los que mantienen una tendencia estable en

\footnotetext{
* GLOBOCAN incluye a los siguientes países: en Sudamérica: Argentina, Bolivia, Brasil, Chile, Colombia, Ecuador, Guyana, Paraguay, Perú, Surinam, Uruguay y Venezuela; en Centroamérica: Belice, Costa Rica, El Salvador, Guatemala, Honduras, México, Nicaragua, Panamá; en El Caribe: Bahamas, Barbados, Cuba, República Dominicana, Haití, Jamaica, Puerto Rico, y Trinidad y Tobago; y en Norteamérica: Canadá y los Estados Unidos de América.
} 


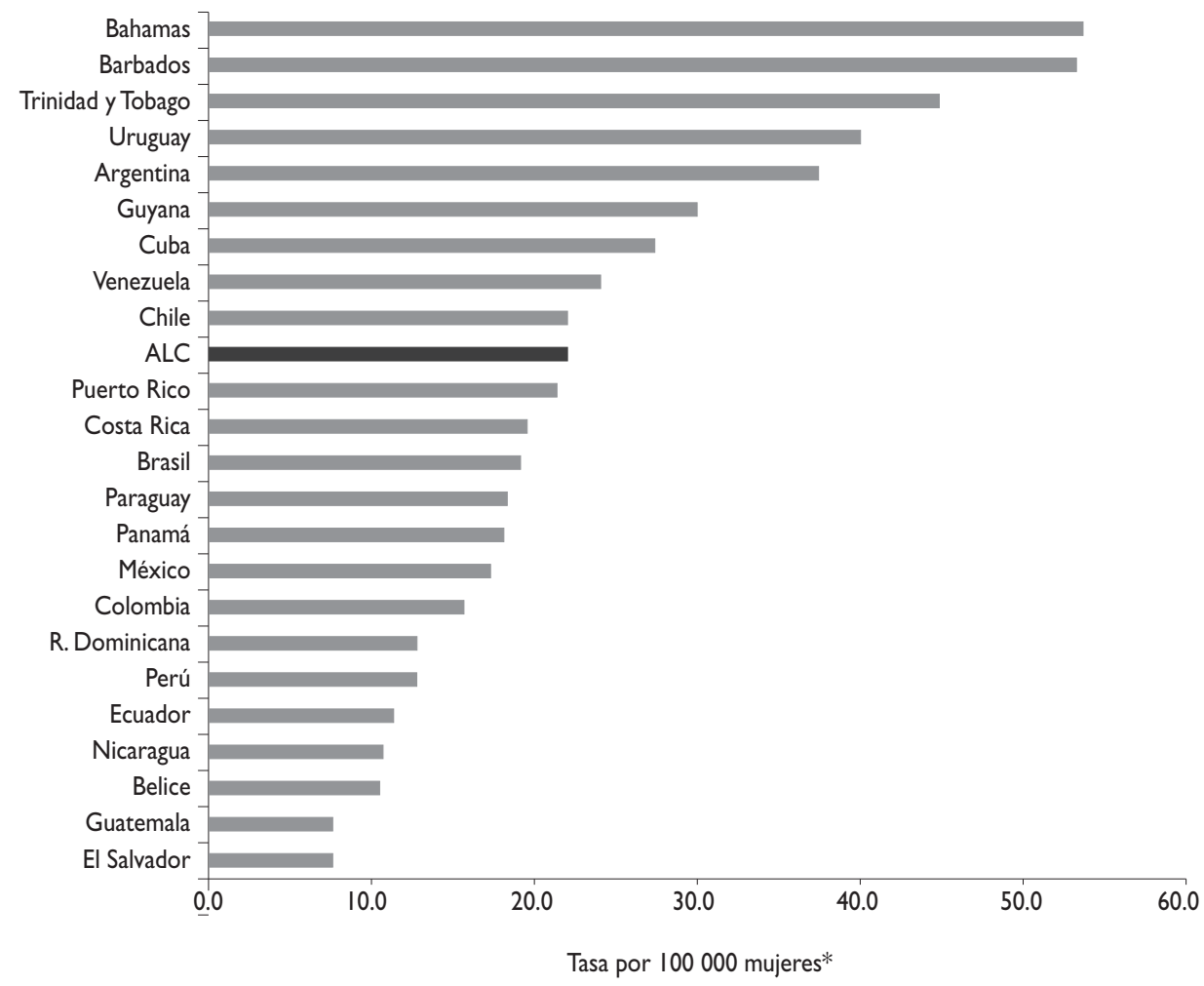

Fuentes: Estadísticas vitales, países seleccionados. Naciones Unidas. Proyecciones de Población 2006.

* Tasas ajustadas por edad.

Figura 2. Mortalidad por cáncer de mama* en mujeres de 25 años y mayores en países seleccionados de América LATINA, 2005

los últimos 20 años (Cuba, Chile, Trinidad Tobago, Barbados); y c) los países en los que aumenta la mortalidad. Destaca el notorio incremento en países como México (84\%) o Venezuela (54\%) y, en menor proporción, Brasil, Costa Rica o Colombia. Al igual que en otras latitudes, en los países centroamericanos el incremento es muy acentuado debido a la baja mortalidad registrada hace 25 años. Varios estudios confirman que la mortalidad en la mayoría de los países latinoamericanos va en aumento, en particular en los países que contaban con una mortalidad relativamente baja, como Colombia, Costa Rica, Ecuador, México y Venezuela. ${ }^{15}$ La tendencia de la mortalidad por CaMa de 1985 a 2005 en países seleccionados de LAC no muestra un decremento claro, si bien se reconoce un grupo de países con alta mortalidad relativamente constante (20 a 50 por 100000$)$ y otro con menor magnitud (menos de 20 por 100000 ), lo que sugiere un incremento de la tendencia (figura $3, \mathrm{~A} \mathrm{y} \mathrm{B}$ ).
$\mathrm{Al}$ ajustar la tendencia de la mortalidad por $\mathrm{CaMa}$ en mujeres de 25 años y mayores se observa que sólo en Uruguay la disminución es estadísticamente significativa. En los otros países en los que la mortalidad desciende, la tendencia no es significativa. En contraste, se observa que en prácticamente todos los países que incrementan su mortalidad la tendencia tiene relevancia estadística (cuadro I).

\section{Razón muertes/casos}

La comparación de las razones de muertes sobre casos de los países del continente americano muestra dos escenarios (figura 4). Si se incluye a Estados Unidos de América y Canadá, la tasa de mortalidad por CaMa y la razón M/C del continente es de 40.4 por 100000 y 0.25, respectivamente. Esto conduce a que todos los países en la región, con excepción de la Unión Americana, se 
desplacen fuera del cuadrante inferior derecho. Por otro lado, si se excluye de la comparación a Canadá y Estados Unidos de América, la tasa de incidencia y la razón de la región son 36.7 por 100000 y 0.34, respectivamente. Esta última comparación permite observar que existen países, como Argentina y Uruguay, que muestran un comportamiento similar al de la Unión Americana, es decir, una mayor incidencia de CaMa y un número relativamente bajo de muertes. La razón de 0.2 refleja una mejor atención médica de la enfermedad a pesar de la detección de muchos casos. En contraste, los países como Bolivia, donde la incidencia de CaMa es relativamente baja y la mortalidad es alta, la razón de
0.47 sugiere menor disponibilidad de recursos médicos para el control de la enfermedad pese a la baja incidencia de casos (figura 4).

\section{Discusión}

Los resultados de este trabajo proporcionan suficiente información para ubicar al CaMa como un problema prioritario de salud pública en LAC y evidencian la necesidad de mejorar la información en cobertura, calidad y oportunidad. Las cifras presentadas sugieren que el CaMa se ha incrementado en incidencia y mortalidad en las mujeres de la región. En muchos
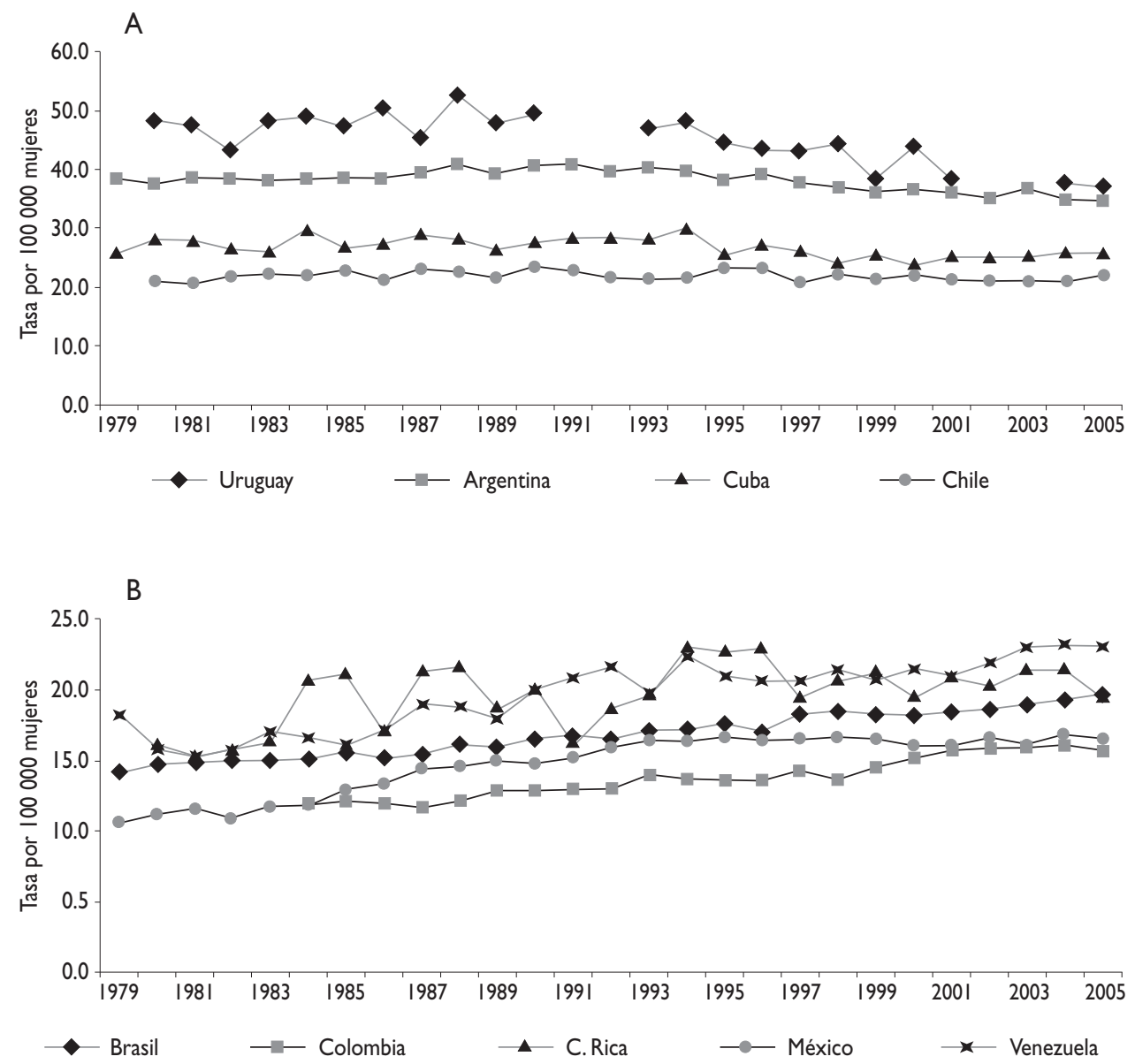

Tasa por 100000 mujeres*

Fuentes: Estadísticas vitales, países seleccionados. Naciones Unidas. Proyecciones de Población 2006

* Tasas ajustadas por edad

Figura 3. Mortalidad por cáncer de mama* en mujeres de 25 años y mayores en países seleccionados de América LATINA, 1979- 2005 
Cuadro I

TENDENCIAS DE MORTALIDAD POR CÁNCER DE MAMA* EN MUJERES DE 25 AÑOS Y MAYORES. Países Seleccionados, 1980-2005

\begin{tabular}{lcccc}
\multicolumn{1}{c}{ País } & $\begin{array}{c}\text { (años en la } \\
\text { serie) }\end{array}$ & $R^{2}$ & Beta & $P$ \\
Argentina & 26 & 0.001 & -0.00036 & 0.91 \\
\hline Bahamas & 15 & 0.000 & -0.00142 & 0.96 \\
\hline Barbados & 20 & 0.09 & 0.276 & 0.19 \\
\hline Belice & 21 & 0.14 & 0.397 & 0.09 \\
\hline Brasil & 25 & 0.96 & 0.197 & $<0.000$ \\
\hline Chile & 25 & 0.02 & -0.0015 & 0.50 \\
\hline Colombia & 21 & 0.93 & 0.217 & $<0.000$ \\
\hline Costa Rica & 25 & 0.31 & 0.167 & 0.003 \\
\hline Cuba & 26 & 0.01 & -0.0154 & 0.62 \\
\hline Ecuador & 26 & 0.72 & 0.134 & $<0.000$ \\
\hline El Salvador & 19 & 0.82 & 0.276 & $<0.000$ \\
\hline Guatemala & 24 & 0.64 & 0.15 & $<0.000$ \\
\hline Guyana & 18 & 0.30 & 0.386 & 0.02 \\
\hline México & 26 & 0.87 & 0.275 & $<0.000$ \\
\hline Nicaragua & 17 & 0.81 & 0.376 & $<0.000$ \\
\hline Panamá & 19 & 0.82 & 0.276 & $<0.000$ \\
\hline Paraguay & 24 & 0.64 & 0.150 & 0.000 \\
\hline Perú & 18 & 0.30 & 0.386 & 0.02 \\
\hline Puerto Rico & 26 & 0.87 & 0.275 & 0.000 \\
\hline R. Dominicana & 23 & 0.28 & 0.0933 & 0.008 \\
\hline Trinidad y Tobago & 25 & 0.12 & 0.264 & 0.09 \\
\hline Uruguay & 20 & 0.31 & -0.242 & 0.009 \\
\hline Venezuela & 26 & 0.89 & 0.335 & $<0.000$ \\
* Tasas ajustadas por edad & & & & \\
\hline & & & & \\
\hline & & & & \\
\hline
\end{tabular}

países ya superó incluso la incidencia y mortalidad del cáncer cervicouterino, como se puede constatar en otras publicaciones. ${ }^{11,15,16}$

Hasta el momento, ningún país en el mundo ha logrado revertir las tendencias crecientes de la incidencia de $\mathrm{CaMa}^{3,17}$ y se observan diferentes patrones en el ritmo de crecimiento que varían de un país a otro. ${ }^{18-19}$ Por ejemplo, mientras que la incidencia aumentó 12\% en Cali, Colombia, de 1973 a 1997, ${ }^{9}$ en Puerto Rico casi se triplicó entre los periodos 1960-64 y 1985-89, ${ }^{20}$ con $68 \%$ de incremento estimado durante los periodos 1973-1977 y 1993-1997, respectivamente. ${ }^{9}$ De manera similar, se advierte un incremento del número de casos confirmados de CaMa que ingresan al Registro Nacional Histopatológico de Cáncer en México, de 9490 en 1998 a 12433 en el 2003.21,22 Es importante aclarar que estas últimas cifras no tienen representatividad nacional ni cuentan con denominadores poblacionales para calcular la incidencia correspondiente.
Con excepción del Reino Unido, Australia, Canadá y Estados Unidos de América, la mortalidad por CaMA no ha disminuido claramente en otros países. ${ }^{14}$ En este último país descendió $2.3 \%$ por año durante el periodo de 1990-2004, ${ }^{23}$ mientras que en el Reino Unido la tasa descendió 31\% entre 1990 y 2006 (de 40.1 defunciones por 100000 mujeres a 27.7 por 100000 mujeres). ${ }^{24}$ Según la OPS, el CaMA es una de las 10 principales causas de muerte en la región. En las mujeres de 30-59 años de edad se ubica en tercer lugar y contribuye con $7.5 \%$ del total de las defunciones de este grupo de edad. ${ }^{25}$ En México se convirtió en la primera causa de muerte por cáncer en mujeres en 2006 y la segunda causa de muerte por todas las enfermedades en mujeres de 30 a 54 años. ${ }^{26}$ En Brasil, el Instituto Nacional de Cáncer refiere que el CaMa es el más incidente en las mujeres de la región del sureste y, sin contar los tumores de la piel, también es el más frecuente en las regiones del sur, centroeste y noreste.

En Estados Unidos de América, las mujeres afroamericanas presentan una incidencia menor y una mortalidad más alta que la población blanca. ${ }^{27}$ Un estudio de base poblacional demuestra que este patrón también se observa en Barbados. En particular, se documenta una elevada mortalidad vinculada con un bajo acceso a mamografía, retraso del diagnóstico oportuno y poco acceso a los tratamientos. ${ }^{28}$ Los datos presentados en este trabajo muestran que a pesar de la baja incidencia (por subregistro $u$ otras razones), en los países más pobres de LAC existe un mayor riesgo de morir por CaMa. Es probable que esto se deba en gran medida a la falta de un diagnóstico oportuno, la escasez de personal capacitado y la falta de acceso a un tratamiento adecuado y oportuno.

Por otra lado, la razón $\mathrm{M} / \mathrm{C}$ puede emplearse como un indicador de las disparidades que existen entre países y sistemas de salud y su capacidad de lograr la detección temprana y ofrecer un diagnóstico y tratamiento oportunos. ${ }^{8,29}$ La razón muertes/ casos es de 0.36 en el plano mundial, para los países en desarrollo es de 0.43 y para las naciones desarrolladas de $0.30 .{ }^{11}$ Una elevada incidencia refleja mejor detección (se identifican más casos) y la mortalidad baja señala un tratamiento adecuado y oportuno (se identifican en fases tempranas con mejor pronóstico). ${ }^{8,29}$ Las regiones con mayor incidencia (Norteamérica, Australia, Europa del Norte, Europa Occidental, Europa del Sur y Sudamérica) cuentan con razones de $\mathrm{M} / \mathrm{C}$ por debajo del promedio mundial de 0.36 . Este patrón demuestra mejores medidas de detección y tratamiento. En la mayoría de las regiones compuestas de países en desarrollo -como África, Asia del Sur, Sureste Asiático, El Caribe y Centroamérica-existe una combinación de mayor mortalidad que la mundial 


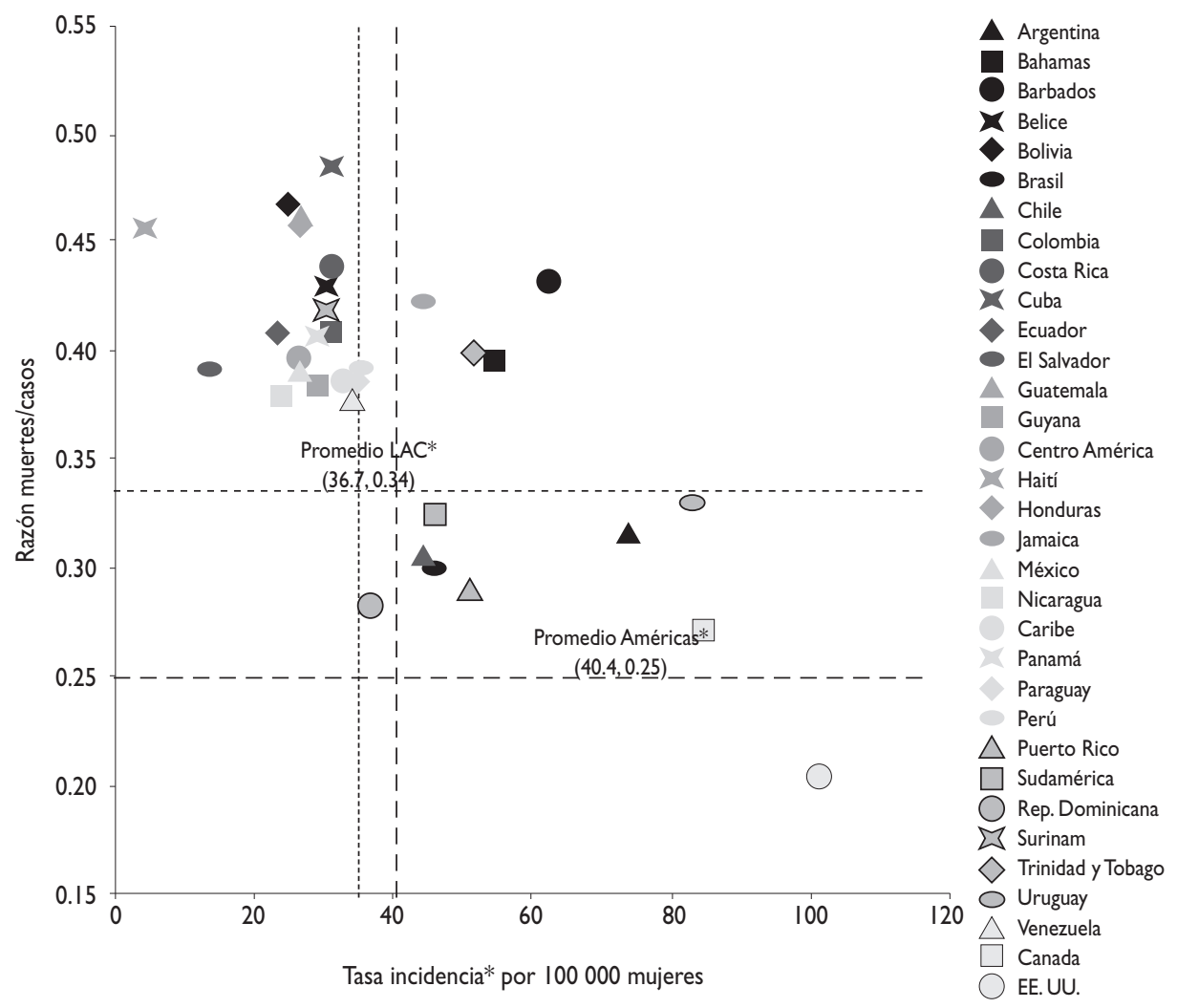

Fuentes: IARC, GLOBOCAN 2002 y referencia 29

*(Tasa de incidencia ajustada por edad, razón $\mathrm{M} / \mathrm{C}$ ), región de LAC

**(Tasa de incidencia ajustada por edad, razón M/C), región de las Américas incluidos Canadá y Estados Unidos

Figura 4. Razón muertes/casos y tasa de incidencia en las Américas, 2002

(razones de 0.39 en El Caribe a 0.71 en África Meridional) a pesar de contar con una menor incidencia. De esta forma, en LAC se identifica un impostergable desafío para los sistemas de salud en materia de detección temprana y un tratamiento adecuado y de calidad, que confronta seriamente el desempeño de los sistemas de salud de la región en aras de mejorar la supervivencia de las mujeres con CaMa.

Cuando se analizan las muertes evitables relacionadas con CaMa en Estados Unidos de América, Reino Unido y Francia se reconoce una tendencia descendente, a diferencia de lo que sucede, en los mismos países, con la diabetes o las enfermedades infecciosas. ${ }^{30} \mathrm{En}$ este sentido, un estudio realizado en México, basado en la propuesta de Nolte ${ }^{31}$ sobre la evaluación del desempeño de los sistemas de salud mediante la atribución categórica de las defunciones por ciertas causas y edades al concepto de "muerte evitable", muestra las disparidades entre los servicios estatales de salud. Este indicador calcula la proporción de muertes evitables con la tecnología disponible para el diagnóstico temprano (mamografía y exploración clínica), los tratamientos (resección, radioterapia y quimioterapia) y la atención médica oportuna y de calidad. Bajo este indicador, $66 \%$ de las defunciones por CaMa que ocurrieron en México entre 2000 y 2004 se pudo evitar. Este índice alcanza 75\% en el Distrito Federal y cerca de $40 \%$ en Campeche. ${ }^{32}$

Es importante considerar la necesidad de incrementar la cobertura, calidad y oportunidad de la información acerca del CaMa en LAC, ya que en el desarrollo de este documento se identificaron algunas deficiencias al respecto. Por ejemplo, la última estimación del IARC o la OMS por países de la región corresponde en promedio al año 2000; por lo tanto, se prefirió calcular las tasas de 
mortalidad más actualizadas con las limitaciones implícitas, en relación con cobertura, oportunidad y calidad de las estadísticas vitales. De acuerdo con el reporte de la OMS, ${ }^{33}$ la mayor parte de los países de la región (58\% de la población) cuenta con registros de calidad y cobertura media; con baja calidad y cobertura se ubican Nicaragua, Paraguay, Perú, Ecuador y República Dominicana (11\% de la población) y con buena calidad figuran Bahamas, Cuba, México, Venezuela y Chile (30\% de la población). Aunque se contaba con datos para más de 30 países, los resultados que se presentaron corresponden a 23 países seleccionados, ya que el resto no disponía de información reciente o con los suficientes años para analizar su tendencia. ${ }^{34}$

Los datos "más recientes" del número de casos de CaMa tienen más de cinco años (p. ej., el último tomo de CI5 se publicó en 2007, pero los datos corresponden a 1998-2002). Con respecto a la información proporcionada por la IARC, las estimaciones del GLOBOCAN se basan en registros del cáncer, los cuales no incluyen a todos los países de LAC, ni son necesariamente representativos de la población del mismo país al que hacen referencia. Los registros del cáncer cubren tan sólo 16\% de la población mundial en relación con la incidencia y $29 \%$ de la población en cuanto a la mortalidad. Los registros que se usan para calcular la incidencia en la serie deCI5 cubren sólo $8 \%$ de la población mundial; ${ }^{17}$ en consecuencia, las tasas notificadas podrían corresponder en mayor proporción a las mujeres con mejor acceso a los servicios y, por lo tanto, subestiman el efecto del CaMa entre las poblaciones más vulnerables ${ }^{6}$ y no explican inconsistencias en cuanto a la tendencia de incidencia, como el caso de Jamaica que notificó un descenso de $8 \%$ de la incidencia del CaMa en el periodo 1988-1997.35,36

Por su parte, la cobertura de los registros en América Latina y El Caribe en 2002 era de 3\% de la población respecto de los datos usados para las tasas de incidencia en $\mathrm{CI} 5,{ }^{19} 10.5 \%$ de la población para las estimaciones de incidencia basadas en todos los registros y $50 \%$ de la población en cuanto a la mortalidad. ${ }^{17}$ En la publicación de CI5 más reciente, ${ }^{18}$ la población mundial representada en las cifras se incrementó de 8\% en 2002 a 11\% en 2007; la de LAC aumentó de 3 a 4\% (23 millones de habitantes). Los únicos países latinoamericanos que cuentan con sitios registrados en IARC son Argentina, Brasil, Chile, Uruguay, Perú, Ecuador, Costa Rica, Honduras, Cuba y Trinidad y Tobago.

Por todo lo anterior, es inaceptable que sean tan pocos los países que cuentan con un registro nacional de cáncer con los estándares que establece la IARC, que permita a los países diseñar mejores políticas de prevención y control del CaMa. Las cifras demuestran que la tendencia del cáncer de mama va en aumento en toda la región y que sin un mejor sistema de información no habrá condiciones para evaluar el impacto de la detección oportuna y el tratamiento adecuado.

\section{Referencias}

I. The global burden of diseases 2004. Update. Geneva:World Health Organization, 2008 [consultado el 7 nov 2008]. Disponible en: http://www. who.int/healthinfo/global_burden_disease/estimates_regional/en/index.html. 2. Coleman M, Quaresma M, Berrino F, Lutz J, De Angelis R, Capocaccia $R$, et al. Cancer survival in five continents: a worldwide population-based study (CONCORD). Lancet Oncol 2008;8(9):730-756.

3. Bray F, McCarron P, Parkin DM. The changing global patterns of female breast cancer incidence and mortality. Breast Cancer Res 2004;6:229-239. 4. Ziegler R, Hoover R, Pike M, Hildesheim A, Nomura A, West DW, et al. Migration patterns and breast cancer risk in Asian-American women.J Natl Cancer Inst 1993;85:1819-1827.

5. López-Ríos O, Lazcano-Ponce EC, Tovar-Guzmán V, Hernández-Avila M. La epidemia de cáncer de mama en México: ¿consecuencia de la transición demográfica? Salud Publica Mex 1997;39:259-265.

6. Porter P."Westernizing” women's risks? Breast cancer in lower-income countries. N Engl J Med 2008;358(3):4.

7. Morimoto T, Nagao T, Okazajy Kira M, Nakagawa Y,Tangoku A. Current status of breast cancer in the world. Jap Breast Can Soc 2009; 16:2-9. 8. Schwartsmann G. Breast cancer in South America: challenges to improve early detection and medical management of a public health problem.J Clin Oncol 200I;15;19(I8s):6.

9. Althuis MD, Dozier JM, Anderson WF, Devesa SS, Brinton LA. Global trends in breast cancer incidence and mortality 1973-1997. Int J Epidemiol 2005;34(2):405-4I2.

10. Shinagawa SM. The excess burden of breast carcinoma in minority and medically underserved communities. Cancer 2000;88(S5):1217-1223. II. Ferlay J, Bray F, Pisani P, Parkin DM. GLOBOCAN 2002: Cancer incidence, mortality and prevalence worldwide. Lyon: IARC Press, 2004. 12. Parkin DM,Whelan SL, Ferlay J, Storm H. Cancer incidence in five continents, Volumes I-VIII. Lyon: IARC Scientific Publications, 2005. 13. Ahmad O, Boschi-Pinto C, Lopez A, Murray CJL, Lozano R, Inoue M. Age standardization of rates. A new WHO standard. GPE Discussion Paper No. 31. Geneva: Organización Mundial de Salud; 2000 [consultado el 7 nov 2008]. Disponible en: http://www.who.int.

14. Garcia M, Jemal A, Ward E, Center M, Hao Y, Siegel R, et al. Global cancer facts and figures 2007. Atlanta, GA:American Cancer Society, 2007. 15. Bosetti C, Malvezzi M, Chatenoud L, Negri E, Levi F, La Vecchia C. Trends in cancer mortality in the Americas, 1970-2000.Ann Oncol 2005; 16(3):489-5II.

16. Phillips AA, Jacobson JS, Magai C, Consedine N, Horowicz-Mehler NC, Neugut Al. Cancer incidence and mortality in the Caribbean. Cancer Invest 2007;25(6):476-483.

17. Parkin DM, Fernández LMG. Use of statistics to assess the global burden of breast cancer. Breast J 2006; I2(Suppl I): 10.

18. Curado MP, Edwards B, Shin HR, Storm H, Ferlay J, Heanue M, et al. Cancer incidence in five continents, Vol. IX. Lyon: IARC Scientific Publications No. 160, 2007.

19. Parkin DM, Whelan SL, Ferlay J, Teppo L, Thomas DB. Cancer incidence in five continents, Vol.VIII. Lyon: IARC Scientific Publications No. I55, 2002. 20. Nazario C, Figueroa-Vallés N, Rosario R. Breast cancer patterns and lifetime risk of developing breast cancer among Puerto Rican females. P R Health Sci J 2000;19(I):7-13.

21. Registro Histopatológico de Neoplasias Malignas. Compendio: mortalidad y morbilidad 1998 [base de datos en Internet]. Secretaría de 
Salud, Epidemiología 1998 [consultado el I5 marzo 2008]. Disponible en: http://www.dgepi.salud.gob.mx/diveent/RHNM.htm.

22. Registro Histopatológico de Neoplasias Malignas. Compendio: mortalidad y morbilidad 200I [base de datos en Internet]. Secretaría de Salud, Epidemiología. 200I [consultado el I5 marzo 2008]. Disponible en: http://www.dgepi.salud.gob.mx/diveent/RHNM.htm.

23. ACS. Breast Cancer Facts \& Figures 2007-2008. Atlanta, GA:American Cancer Society, 2007.

24. Trends in breast cancer mortality. London: Cancer Research UK; 2008 [updated 2008; consultado el 6 agosto 2008]; Disponible en: http://info. cancerresearchuk.org/cancerstats/types/breast/mortality/.

25. Chapter 2. Health conditions and trends. En: Health in the Americas, 2007. Washington, DC: Organización Panamericana de la Salud, 2007:58207.

26. Knaul F, Nigenda G, Lozano R,Arreola-Ornelas H, Langer A, Frenk J. Breast cancer in Mexico: a pressing priority Reprod Health Matters 2008;16(32):I-II.

27. Smigal C, Jemal A, Ward E, Cokkinides V, Smith R, Howe HL, et al. Trends in breast cancer by race and ethnicity: Update 2006. CA Cancer J Clin 2006;56(3): 168-183.

28. Hennis A, Hambleton I,Wu S, Leske M, Nemesure B. Breast cancer incidence and mortality in a Caribbean population: Comparisons with
African-Americans. Int ] Cancer 2008;|23:1097-20I5.

29. López-Carrillo L. Capítulo I7. El cáncer mamario: epidemiología y prevención. En:Tamayo RP, ed. El cáncer en México. México, DF: El Colegio Nacional, 2003:443-56.

30. Nolte E, McKee M. Measuring the health of nations: Updating an earlier analysis. Health Affairs 2008;29(I):58-7I.

3I. Nolte E, McKee M. Measuring the health of nations:Analysis of mortality amenable to health care. BMJ 2003;327: I | 29- I I 33.

32. Franco-Marina F, Lozano R, et al. La mortalidad en México, 20002004: Muertes evitables: magnitud, distribución y tendencias México, DF: Secretaría de Salud, 2006.

33. Mathers C, Ma Fat D, Inoue M, Rao C, Lopez A. Counting the death and what they died from: an assessment of the global status of cause of death data. Bull World Health Organ 2005;83(3): I7I-I77.

34.WHO mortality database, varios años. World Health Organization; [consultado el 7 nov 2008]; Disponible en: http://www.who.int/healthinfo/ morttables/en/index.html.

35. Hanchard B, Blake G, Wolff C, Samuels E, Waugh N, Simpson D, et al. Age-specific incidence of cancer in Kingston and St. Andrew, Jamaica, 1993-1997. West Indian Med J 2001;50(2):123-129.

36. Brooks S, Hanchard B, Wolff C, Samuels E, Allen J.Age-specific incidence of cancer in Kingston and St. Andrew, Jamaica, 1988-1992. West Indian Med J 1995;44(3): 102-105. 